\title{
L'appropriation des NTIC par les PME
}

\author{
Lahcen Darhouani ${ }^{1}$ \\ Professionnel de recherche
}

\section{Introduction}

L'appropriation des nouvelles technologies d'information et de communication, (NTIC) apparaît comme un cheminement innovateur et irréversible qui impose la restructuration des institutions, des organisations et des territoires. La généralisation des NTIC se confirme comme un phénomène porteur d'un avenir meilleur, forçant les institutions à faire des efforts et les organisations à développer des stratégies ad hoc. Des changements liés à ces stratégies et aux dynamiques qui en résultent sont observables partout dans nos sociétés et prennent des dimensions jamais égalées. Ces changements dépendent d'une double articulation. D'une part, l'appropriation des NTIC fournit une base nouvelle aux activités économiques et sociales en place et, en ce sens, favorise le renouvellement des pratiques entrepreneuriales. D'autre part, cette appropriation fournit aux collectivités et aux individus de meilleures occasions, qui peuvent se traduire par l'innovation dans la mise en place de diverses stratégies de développement. Bien que cette double articulation s'inscrive dans la sphère du renouveau économique et socioculturel, elle donne des vocations nouvelles aux technologies qui inspirent les attitudes et les comportements des acteurs individuels et collectifs.

Afin de mieux comprendre l'ampleur des transformations qu'apporte l'usage des nouvelles technologies dans les entreprises québécoises, nous avons tenté de mesurer plusieurs indicateurs. Les résultats sont présentés selon les méthodes statistiques descriptives. Les données sont comparées en pourcentage. Ces résultats et données confirment que les entreprises, quelle que soit leur région d'implantation, s'adaptent de manière inégale à l'avènement et au développement des technologies. Bien que les résultats que nous présentons ici montrent que la disparité entre les territoires prend des dimensions nouvelles, une analyse plus approfondie serait utile à qui veut saisir l'ampleur des changements occasionnés par l'innovation technologique.

\section{Ces changements dépendent d'une double articulation. D'une part, l'appropriation}

des NTIC fournit une base nouvelle aux activités économiques et sociales en place et, en ce sens, favorise le renouvellement des pratiques entrepreneuriales. D'autre part, cette appropriation fournit aux collectivités et aux individus de meilleures occasions, qui peuvent se traduire par l'innovation dans la mise en place de diverses stratégies de développement. Bien que cette double articulation s'inscrive dans la sphère du renouveau économique et socioculturel, elle donne des vocations nouvelles aux technologies qui inspirent les attitudes et les comportements des acteurs individuels et collectifs.

\section{Contexte de la recherche}

L'objectif de l'enquête présentée ici était de saisir comment cette nouveauté et ce développement s'opèrent dans quatre territoires du Québec: les régions de Québec, du Saguenay, de Manicouagan et de Rivière-du-Loup. L'enquête a permis de saisir le degré d'appropriation du matériel informatique et des logiciels, et d'en connaître les effets sur le traitement de l'information.

Le questionnaire qui servit à la collecte de données comprend trois axes. Le premier concerne le profil des entreprises (nombre de places d'affaires, nombre d'employés, âge des entreprises, chiffre d'affaires et sources de concurrence). Le deuxième axe est réservé aux besoins, à la demande et à la circulation de l'information. L'accent est mis sur les sources d'information, les moyens d'interac- 
tion, le type d'information, les activités à l'extérieur de l'organisation, la participation à des réseaux d'entreprises, les systèmes de communication et les systèmes de classement de l'information. Le troisième axe traite de l'innovation technologique. On s'y intéresse à l'usage que l'on fait du matériel informatique et d'un nombre de logiciels, de même que sur le branchement des entreprises à l'Internet et à son utilisation.

La collecte des données s'est déroulée entre juillet et décembre 2000. Le questionnaire a été envoyé à 290 entreprises préalablement contactées par téléphone. $\mathrm{Ce}$ questionnaire a suscité chez les entreprises des réactions diverses, certaines de la région de Québec refusant d'y collaborer en prétextant être trop sollicitées par les chercheurs. De plus, nous avons rejeté 13 cas qui présentaient des lacunes importantes, ce qui ne permettait pas de les inclure dans notre étude. Au total, notre échantillon est donc formé de 132 questionnaires contenant des données qui se prêtent bien à l'interprétation. Il s'agit d'un taux de réponse de 45,5\%. Nous avons reçu les derniers questionnaires fin décembre 2000 et début 2001.

La compilation et le traitement des données ont été réalisés avec le logiciel Statistical Package for the Social Science, SPSS. Ce logiciel fournit des outils statistiques qui ont servi à comparer les données portant sur les quatre territoires. Nous avons utilisé la version 10.0 pour Windows de ce logiciel.

Les paragraphes qui suivent montrent les caractéristiques générales des PME qui constituent notre échantillon. L'accent est mis sur la répartition des PME selon le territoire de leur appartenance, le nombre de places d'affaires, le nombre d'employés, le nombre d'années d'opération, le chiffre d'affaires et la source de concurrence.
La répartition selon leur région d'implantation des entreprises constituant notre échantillon montre que $45,5 \%$ de ces organisations sont localisées au Saguenay. Seulement $10 \%$ d'entre elles sont implantées à Rivière-du-Loup. Dans la région de Québec, les entreprises représentent 26\% de l'échantillon, comparativement à 19\% à Manicouagan.

Figure 1: Répartition des entreprises de l'échantillon selon le territoire de leur appartenance, en \%

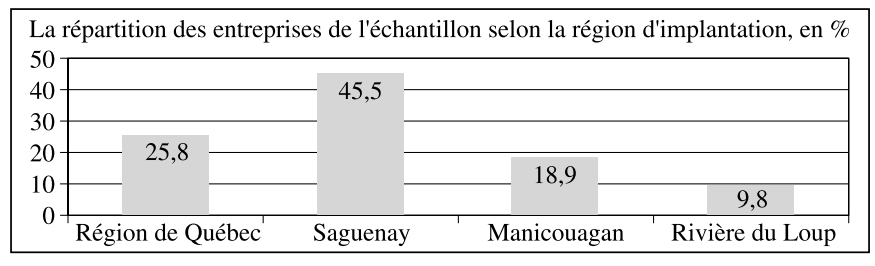

La distribution des entreprises par nombre de places d'affaires montre que $61 \%$ de notre échantillon a une seule place d'affaires, alors que les entreprises ayant plus d'une place d'affaires représentent $40 \%$ de ce même échantillon.

Figure 2: Répartition des entreprises selon le nombre de places d'affaires, en $\%$

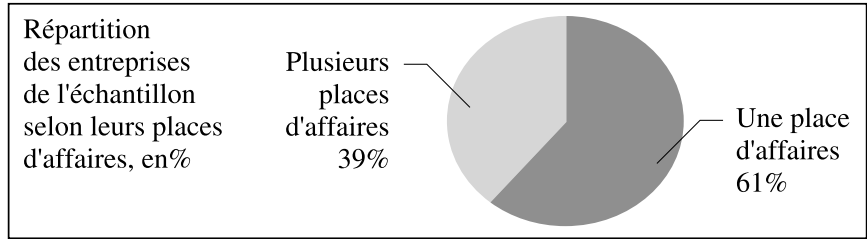

La distribution de l'échantillon selon le nombre d'employés montre que $76,5 \%$ des entreprises ont 50 employés et moins, et $22 \%$ ont plus de 50 employés. De même, $38 \%$ des entreprises de notre échantillon sont actives depuis moins de 10 ans, alors que 54\% ont 13 ans d'activité et plus, dont $36 \%$ ont plus de 16 ans d'activité.

\section{Figure 3: Répartition des entreprises selon le nombre d'employés, en \%}

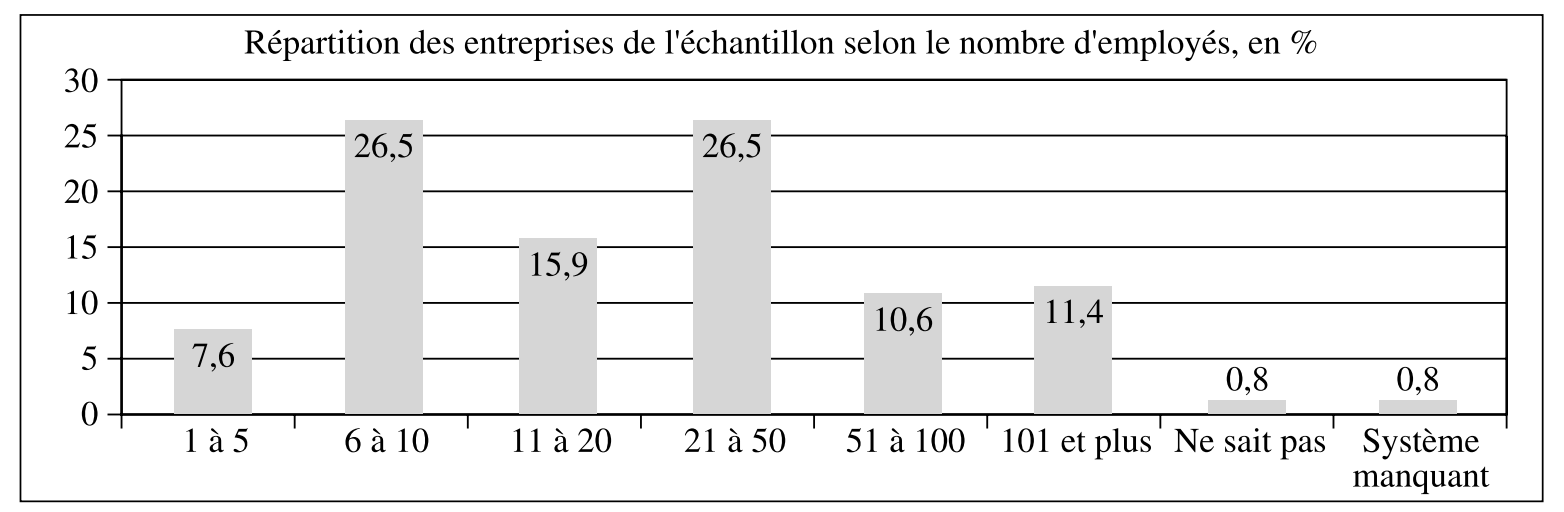


Figure 4 : Répartition des entreprises selon le nombre d'années d'opération, en \%

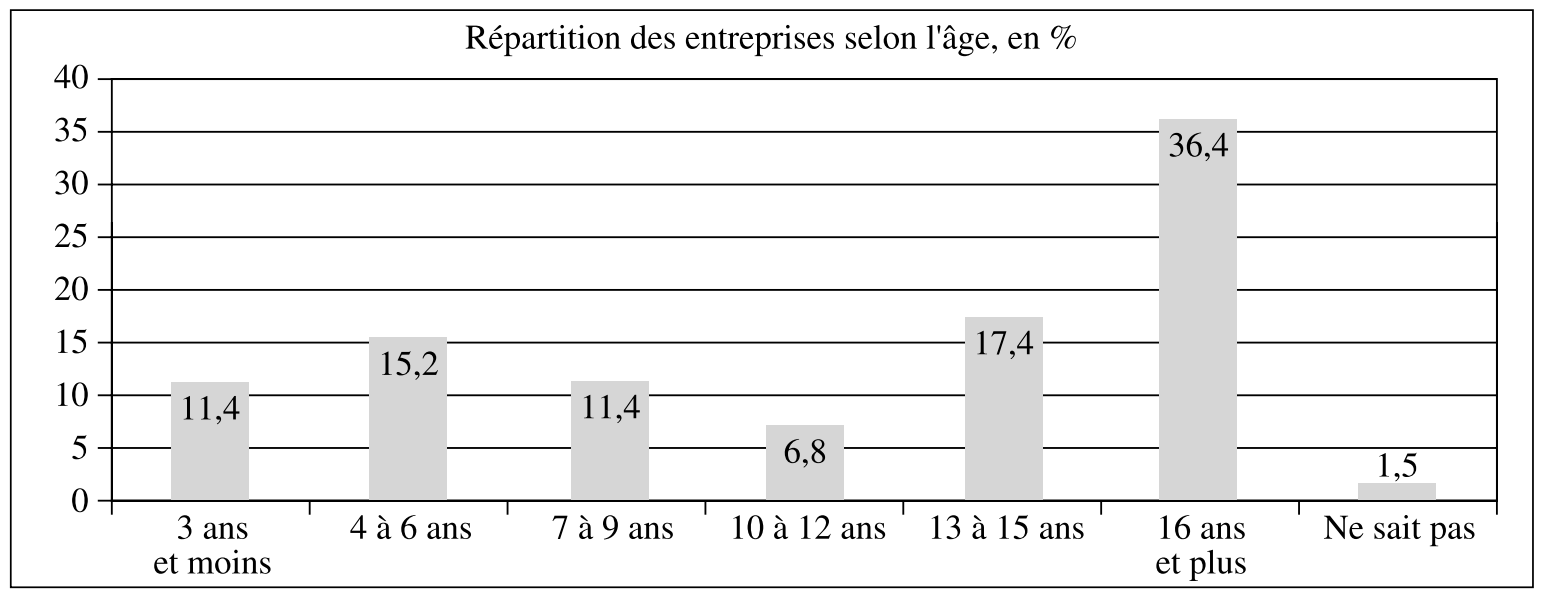

De plus, la répartition de notre échantillon selon le chiffre d'affaires est rapportée à la figure 5. Les entreprises dont le chiffre d'affaires se situe entre 2000001 et $2500000 \$$ représentent $24 \%$ de notre échantillon, $17 \%$ ont un chiffre d'affaires qui ne dépasse pas $5000000 \$$ alors que $15 \%$ ont un chiffre d'affaires de $5000000 \$$ et plus.

\section{Figure 5 : Répartition des entreprises selon le chiffre d'affaires, en \%}

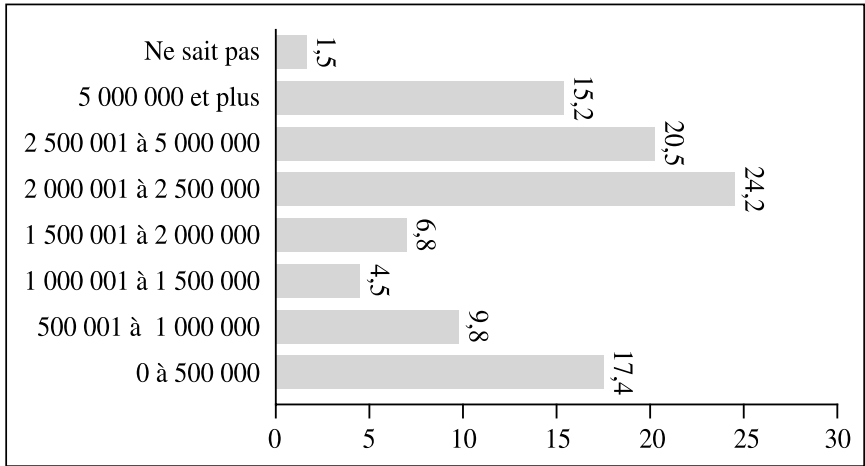

La répartition des PME de notre échantillon permet de voir que $41 \%$ des entreprises de la région de Québec participent à des réseaux d'entreprise. Ce pourcentage est plus élevé que ceux enregistrés à Manicouagan (24\%) et à Rivière-du-Loup (15\%), mais inférieur à celui du Saguenay ( voir la figure 6).

Enfin, la distribution de notre échantillon selon la source de concurrence montre que $30 \%$ des entreprises mènent une concurrence locale ${ }^{2}, 23 \%$ font face à une concurrence régionale, $26,5 \%$ mènent une concurrence au niveau international et $18 \%$ seulement, au niveau national.
Figure 6: L'affiliation des entreprises à des réseaux

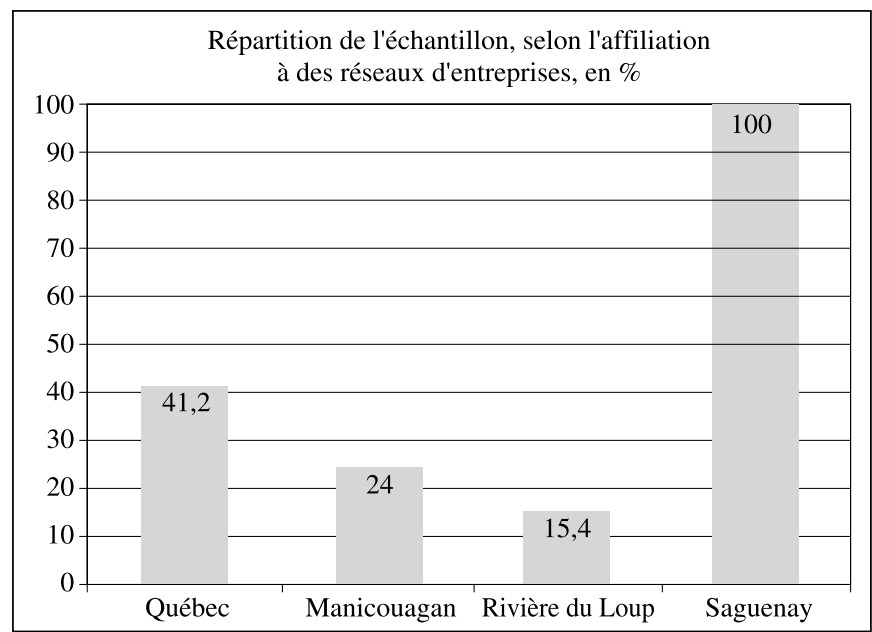

Figure 7 : Répartition des entreprises selon les sources de concurrence, en \%

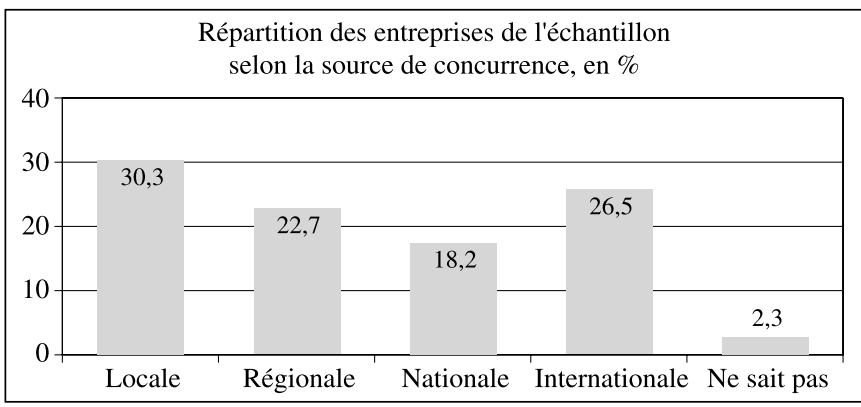

À la lumière des caractéristiques des PME constituant notre échantillon, nous essayerons donc de présenter les axes du questionnaire qui a servi à recueillir les données empiriques. 


\section{L'information: besoins, demande et circulation}

Dans le cadre de cette enquête, nous avons voulu connaître les besoins, la demande et la circulation de l'information. Ensemble, ces trois axes sont souvent associés au terme «informationnel» désignant «une forme particulière d'organisation sociale, dans laquelle la création, le traitement et la transmission de l'information deviennent les sources premières de la productivité et du pouvoir, en raison des nouvelles conditions technologiques apparaissant dans cette période historique-ci ${ }^{3} \gg$. L'information occupe une place centrale dans les dynamiques économiques et sociales. Elle suggère à chaque territoire sa propre logique de développement car un mode de développement singulier est souvent associé à une dynamique particulière.

En effet, «chaque mode de développement dispose [...] d'un principe d'efficacité structurellement déterminé, autour duquel s'organisent les processus technologiques ${ }^{4} \gg$. Nous tentons de cerner le mode de développement, dans ce cas-ci à partir d'un «informationnalisme (qui) vise au développement technologique. C'est-à-dire à l'accumulation de savoir et à la complexité croissante du traitement de l'information. Si un accroissement du savoir peut normalement entraîner une augmentation du rendement par unité de production, c'est la recherche de savoir et d'information qui, dans le mode informationnel, caractérise la fonction technologique de production ${ }^{5} »$.

Nous avons cherché à obtenir des données sur les sources que les entrepreneurs utilisent pour recueillir l'information stratégique. Nous nous sommes également intéressés aux moyens d'interaction, aux types d'information qui intéresse les entrepreneurs et aux activités auxquelles les entrepreneurs assistent à l'extérieur de leurs organisations. Dans les sections suivantes, nous examinons de plus près l'ensemble de ces données.

\subsection{Les sources d'informations économiques}

On a cherché à connaître les principales sources d'information que les entrepreneurs utilisent dans leurs activités professionnelles, à l'aide de la question suivante: Quelles sources d'informations économiques considérezvous les plus utiles dans vos activités professionnelles?

Les sources utilisées très souvent sont les clients par $68 \%$, les contacts personnels (47\%) et les fournisseurs (42\%).
Les sources les moins utilisées sont les centres de recherche : $25 \%$ des entreprises ne les utilisent jamais et $34 \%$ d'entre elles ne recourent jamais aux universités. Dans notre échantillon, 23\% des entreprises n'utilisent jamais les services de sociétés de développement, $28 \%$ ne sollicitent jamais de sociétés-conseil, 26,5\% n'ont jamais visité d'entreprises canadiennes et $17 \%$ n'ont jamais utilisé les services fédéraux. Par ailleurs, les sources d'information utilisées souvent sont les fournisseurs, par $34 \%$ des entreprises, les centres de recherche et les sociétés-conseils, par $22 \%$, le gouvernement fédéral, par $30 \%$, le gouvernement provincial, par 32\%. Parmi les entreprises, $42 \%$ consultent des journaux et revues spécialisés, $33 \%$, des revues et journaux régionaux et un même pourcentage est rapporté dans le cas des foires et expositions.

Un nombre important d'entreprises fait appel peu souvent ou rarement à des sources extérieures d'information. Ainsi, $42 \%$ des entreprises sollicitent peu les centres de recherche, $48 \%$, les universités, $40 \%$, le gouvernement fédéral et enfin, $36 \%$ d'entre elles font rarement appel aux services du gouvernement provincial. Dans le cas de visites d'entreprises à l'étranger, il y a faible utilisation par $38 \%$ de notre échantillon soit, pour les sociétésconseil, $40 \%$, pour les sociétés de développement, $45 \%$, pour les foires et expositions, $35 \%$ et finalement, pour les visites d'entreprises canadiennes, 47\%. D'un autre côté, le recours à plusieurs sources d'information montre une différence significative entre les quatre territoires. Par exemple, le pourcentage des entreprises qui recourent très souvent et souvent aux fournisseurs pour s'informer représente 38\% dans la région de Québec et au Saguenay alors que $44 \%$ des entreprises implantées à Manicouagan s'informent très souvent auprès des fournisseurs et que $61,5 \%$ le font à Rivière-du-Loup. De même, $62 \%$ des entreprises de la région de Québec s'informent très souvent auprès des clients. Le pourcentage est de $70 \%$ au Saguenay, de $60 \%$ à Manicouagan et atteint $92 \%$ à Rivière-du-Loup. Quant aux centres de recherche et aux universités, on constate une autre réalité: $26,5 \%$ des entreprises de la région de Québec ne s'informent jamais auprès des centres de recherche et $29 \%$, auprès des universités. Ces deux pourcentages sont respectivement de $17 \%$ et de $23 \%$ au Saguenay. Ils sont de $40 \%$ et de $52 \%$ à Manicouagan et de $31 \%$ et de $54 \%$ à Rivière-du-Loup.

L'ensemble de ces données montre que les sources d'information sont diversifiées d'un territoire à l'autre. Les besoins d'information ne sont pas les mêmes partout, 
variant non seulement en fonction des dynamiques économiques et sociales de chaque territoire mais également en fonction de la taille des entreprises. Ainsi, les sources d'information que les entreprises utilisent le plus sont les clients, les contacts personnels, les fournisseurs et les journaux et revues spécialisées. Les sources que ces entreprises négligent sont les centres de recherche, les universités, les sociétés-conseils, les sociétés de développement et les visites des entreprises canadiennes. De même, les entreprises implantées à Rivière-du-Loup et à Manicouagan comptent davantage sur les fournisseurs et les clients que celles qui sont implantées dans la région de Québec et à Saguenay. Enfin, les entreprises implantées à Rivière-du-Loup et à Manicouagan consultent plus les universités et les centres de recherche que celles qui sont implantées dans la région de Québec et à Saguenay.

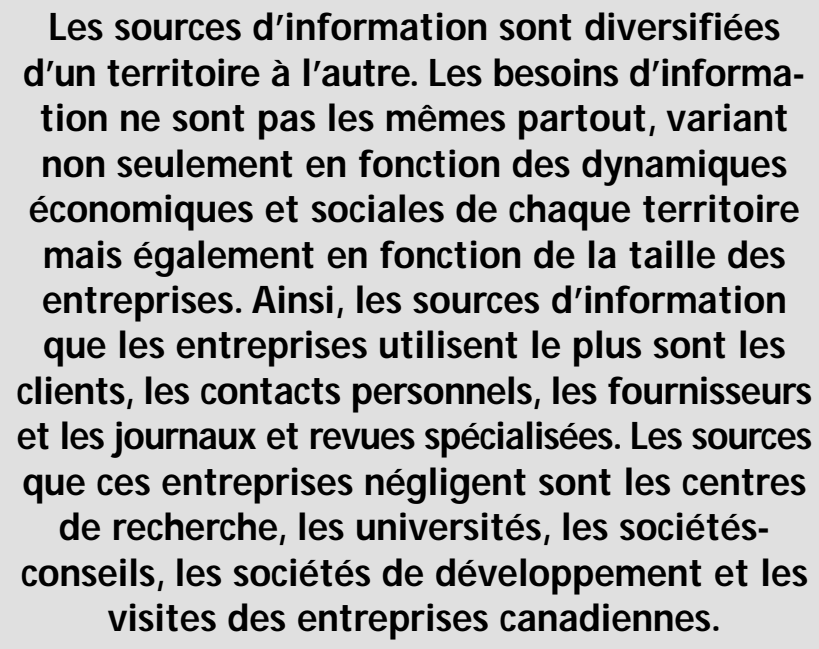

\subsection{Les moyens d'interaction}

Une partie de notre enquête a été consacrée aux moyens que les entrepreneurs utilisent dans leurs interactions avec les autres organisations. La question posée était: Quels sont vos moyens d'interaction (en nombre) avec les autres organisations dans une semaine? La formulation de cette question est orientée par la logique qui sous-tend l'usage que l'on fait des composantes techniques et technologiques à l'occasion des activités quotidiennes. Les réponses à cette question permettent de mieux comprendre «la convergence et l'interaction entre un nouveau paradigme technologique et une nouvelle logique organisationnelle qui constituent le fondement historique de l'économie informationnelle ${ }^{6} \gg$. Ainsi, on s'intéressait dans l'enquête à des questions sur l'usage que l'on fait du téléphone, de la télécopie, des activités de face-à-face, de la place publique et du courriel. Comme on peut le constater, il s'agit bien de moyens traditionnels (face-àface et place publique) et d'autres, plus modernes (le téléphone, la télécopie et le courriel). Ces questions concernent l'usage de tous ces moyens pour recevoir et pour émettre l'information stratégique. Les réponses montrent que le téléphone est le moyen le plus utilisé par les dirigeants : $74 \%$ des entreprises l'utilisent pour recevoir l'information 20 fois et plus par jour et 70,5\% l'utilisent à la même fréquence pour émettre des messages. Ces deux pourcentages sont respectivement de $64 \%$ et $62 \%$ dans le cas de la télécopie et de 50 et $45,5 \%$ pour ce qui est du courriel. De plus, la place publique est un moyen d'interaction que l'on utilise moins de cinq fois par jour dans $69 \%$ des entreprises. De même, 20,5\% de celles-ci recourent aux activités de face-à-face pour recevoir l'information stratégique moins de 5 fois par jour et $20 \%$ l'utilisent à la même fréquence pour émettre l'information.

Ces pourcentages concernant tout notre échantillon cachent une réalité qui varie d'un territoire à l'autre. Par exemple, 59\% des entreprises de la région de Québec utilisent le téléphone plus de 20 fois par jour pour recevoir de l'information et 50\% l'utilisent à la même fréquence pour l'émettre. Ces pourcentages sont respectivement de $72 \%$ et $68 \%$ à Manicouagan. Ils sont de $88 \%$ et de $86 \%$ au Saguenay et de $53,5 \%$ et de $54 \%$ à Rivière-du-Loup.

Quant au rôle de la place publique pour recevoir ou émettre de l'information, 68\% des entreprises de la région de Québec y recourent moins de 5 fois par jour pour en recevoir et $65 \%$ aussi peu souvent pour en diffuser. Ces deux pourcentages sont moins importants que ceux rapportés au Saguenay, soit 78\% dans les deux cas. Ces mêmes pourcentages sont respectivement de $60 \%$ à Manicouagan et de $46 \%$ à Rivière-du-Loup. Seulement 3\% des entreprises de la région de Québec utilisent la place publique pour recevoir et émettre l'information 20 fois et plus par jour. Le pourcentage est de $8 \%$ à Manicouagan, $3 \%$ au Saguenay et $8 \%$ à Rivière-du-Loup.

Généralement, les entreprises utilisent très souvent le téléphone et la télécopie pour émettre ou recevoir l'information et elles utilisent moins les activités de face-à-face et la place publique. Le téléphone et la télécopie sont plus utilisés au Saguenay et à Manicouagan que dans la région de Québec et à Rivière-du-Loup. 


\section{Généralement, les entreprises utilisent très souvent le téléphone et la télécopie pour émettre ou recevoir l'information et elles utilisent moins les activités de face-à-face et la place publique.}

\subsection{Types d'information}

Dans l'enquête, on voulait savoir le type d'information que les dirigeants des entreprises cherchent à recueillir. La question posée était la suivante: De quel type d'information avez-vous besoin dans vos activités professionnelles? Les résultats montrent que l'information recherchée est variable. Ainsi, l'information la plus recherchée concerne les nouveaux produits, la concurrence, les technologies, les techniques et les modalités de gestion. De notre échantillon, $40 \%$ des entreprises cherchent très souvent de l'information sur les nouveaux produits. Celles qui cherchent aussi très souvent l'information sur la concurrence représentent $31 \%$. Ce pourcentage est de $33 \%$ en ce qui concerne l'information technologique et $29,5 \%$ dans le cas de l'information technique.

Par ailleurs, $38 \%$ des entreprises sondées ne cherchent jamais d'information sur la sécurité, 23,5\% ne cherchent jamais d'information scientifique et $17 \%$, d'information juridique. L'information que l'on cherche souvent concerne la concurrence (39\%), les nouveaux produits (47\%), la technologie (44\%), la technique (45\%), les ressources publiques $(31 \%)$ et l'information sur les modalités de gestion $(40 \%)$.

Ces données varient substantiellement d'un territoire à l'autre. Ainsi, 44\% des entreprises de la région de Québec cherchent peu souvent de l'information sur la conjoncture et seulement $15 \%$ la cherchent très souvent. Les pourcentages sont respectivement de $47 \%$ et de $10 \%$ au Saguenay; ils sont de $23 \%$ et de $31 \%$ à Rivière-du-Loup. Le pourcentage des entreprises qui cherchent peu souvent cette information est de 32\% à Manicouagan.

De plus, 56\% des entreprises de la région de Québec cherchent très souvent de l'information sur la concurrence. Cette même catégorie représente $46 \%$ à Rivièredu-Loup, 36,7\% au Saguenay et $16 \%$ à Manicouagan. $\mathrm{Au}$ Saguenay, $48 \%$ des entreprises cherchent souvent cette même information sur la concurrence. Ce pourcentage est comparable à celui que nous avons enregistré à Rivière-du-Loup (46\%) mais il est un peu plus élevé que celui rapporté dans les cas de Manicouagan (28\%) et de Québec (29\%).

Certaines sources d'information sont plus sollicitées à Rivière-du-Loup, comparativement aux autres territoires. Ainsi, 54\% des entreprises implantées dans ce territoire cherchent très souvent de l'information sur les nouveaux produits. Cette catégorie représente $50 \%$ dans la région de Québec et $40 \%$ au Saguenay. Ce même pourcentage ne dépasse pas $20 \%$ à Manicouagan. De plus, $31 \%$ des entreprises de la région de Rivière-du-Loup cherchent très souvent de l'information sur les exportations contre $27 \%$ au Saguenay et $12 \%$ dans la région de Québec.

\section{Les entreprises cherchent plus l'information concernant les nouveaux produits, la concurrence, l'information technologique et technique ainsi que l'information portant sur les modalités de gestion. Les entreprises s'intéressent moins à l'information concernant la sécurité, l'information scientifique et l'information juridique.}

En général, les entreprises cherchent plus l'information concernant les nouveaux produits, la concurrence, l'information technologique et technique ainsi que l'information portant sur les modalités de gestion. Les entreprises s'intéressent moins à l'information concernant la sécurité, l'information scientifique et l'information juridique. Comparativement aux entreprises implantées à Manicouagan et à Rivière-du-Loup, les entreprises de Québec et du Saguenay cherchent moins l'information sur la conjoncture et la concurrence. De même, les entreprises implantées à Rivière-du-Loup et à Québec s'intéressent davantage à l'information concernant les nouveaux produits et les exportations.

\subsection{Les activités à l'extérieur}

La question suivante concernait les activités en dehors de l'entreprise: Participez-vous à des activités à l'extérieur de votre milieu de travail? Les résultats montrent que les dirigeants de ces entreprises participent à de nombreuses activités extérieures. Les repas sont une occasion préférée par un grand nombre: $23,5 \%$ d'entre eux y participent très souvent, $38 \%$ y participent souvent, $29,5 \%$ y participent peu souvent et seulement $7 \%$ n'y participent jamais. Dans le cas des expositions, ces 
mêmes pourcentages sont respectivement de $8 \%, 36 \%$, $48 \%$ et $8 \%$.

Par ailleurs, 38\% de ces dirigeants ne participent jamais aux activités caritatives et $36 \%$ y participent peu souvent. Ces deux pourcentages sont respectivement de $23,5 \%$ et $51,5 \%$ dans le cas des événements mondains. Ces mêmes pourcentages sont de $17 \%$ et de $49 \%$ pour ce qui est des événements spéciaux. Ils sont aussi, respectivement, de $23,5 \%$ et de $4 \%$ dans le cas des activités de reconnaissance et de $35 \%$ et de $45 \%$ dans le cas des activités liées à l'environnement.

Si ces données montrent que le pourcentage des entreprises qui participent à diverses activités extérieures varie selon le type d'activité, ces données cachent cependant des écarts significatifs entre les territoires.

Pour ce qui est des activités caritatives, un fort pourcentage n'y participe jamais, $44 \%$ dans la région de Québec, $46 \%$ à Rivière-du-Loup, 33\% au Saguenay et $36 \%$ à Manicouagan. Par ailleurs, 28\% des entreprises du Saguenay participent très souvent à des repas. Ce pourcentage est de $26,5 \%$ dans la région de Québec et de $20 \%$ à Manicouagan. Ceux qui y participent souvent représentent 54\% à Rivière-du-Loup, 38\% au Saguenay, 36\% à Manicouagan et $32 \%$ dans la région de Québec. En général, on peut dire que l'ensemble des données montre que les dirigeants des entreprises des différents territoires participent inégalement aux différentes activités organisées à l'extérieur de leur organisation.

Les dirigeants d'entreprises s'intéressent davantage aux repas, aux activités caritatives et aux activités de reconnaissance. Un pourcentage important des dirigeants s'intéresse moins aux expositions et ne voit pas l'utilité des activités caritatives.

Les dirigeants d'entreprises s'intéressent davantage aux repas, aux activités caritatives et aux activités de reconnaissance. Un pourcentage important des dirigeants s'intéresse moins aux expositions et ne voit pas l'utilité des activités caritatives. Comparativement avec Québec et Saguenay, les dirigeants des entreprises implantées à Manicouagan et à Rivière-du-Loup participent davantage aux repas.

\subsection{L'affiliation à des réseaux d'entreprises}

Une question importante portait sur l'affiliation des entreprises à des réseaux inter-organisationnels. La question était la suivante: Votre entreprise participe-t-elle à des réseaux d'entreprises (trois entreprises ou plus)? Dans ces réseaux, des directeurs, des administrateurs ou les entreprises elles-mêmes sont les principaux acteurs considérés. On cherche donc à comprendre si ces acteurs s'impliquent dans des rapports plus formels de collaboration. Ordinairement, cette implication facilite l'acquisition d'information stratégique et stimule l'échange de certains services. Elle permet à ces mêmes acteurs de mieux se préparer collectivement à affronter les imprévus de conjonctures changeantes.

En général, les réseaux inter-organisationnels se distinguent par des rapports de coordination et de coopération, mais aussi de concurrence. De ce point de vue, l'échange d'information, de savoir et de savoir-faire constitue une sorte de plate-forme commune, favorable à une prise en charge collective et à la mise en place d'un ensemble de standards, adaptés aux besoins du développement local comme à ceux du marché.

En général, les réseaux inter-organisationnels se distinguent par des rapports de coordination et de coopération, mais aussi de concurrence. De ce point de vue, l'échange d'information, de savoir et de savoir-faire constitue une sorte de plate-forme commune, favorable à une prise en charge collective et à la mise en place d'un ensemble de standards, adaptés aux besoins du développement local comme à ceux du marché.

Quoi qu'il en soit, la réponse à cette question a permis de constater que $36 \%$ des entreprises participent à des réseaux inter-organisationnels et $62 \%$ n'y participent pas. Ces pourcentages masquent les réalités fort différentes des quatre territoires. En effet, $41 \%$ des entreprises de la région de Québec participent à ces réseaux. Ce pourcentage est plus élevé que celui enregistré à Manicouagan (24\%) et à Rivière-du-Loup (15\%), mais inférieur à celui du Saguenay.

\subsection{Les systèmes de communication interne}

Les systèmes de communication constituent un moyen par lequel les dirigeants d'entreprises facilitent la circu- 
lation de l'information à l'intérieur de leurs organisations. Souvent, on utilise ces systèmes pour rejoindre l'ensemble du personnel. Chaque organisation choisit un ou plusieurs systèmes qui sont mieux adaptés à ses besoins. À cet égard, nous avons posé la question suivante: Avez-vous un système de communication à l'intérieur de l'entreprise?

Figure 8: Les systèmes de communication adoptés par les entreprises, tout l'échantillon

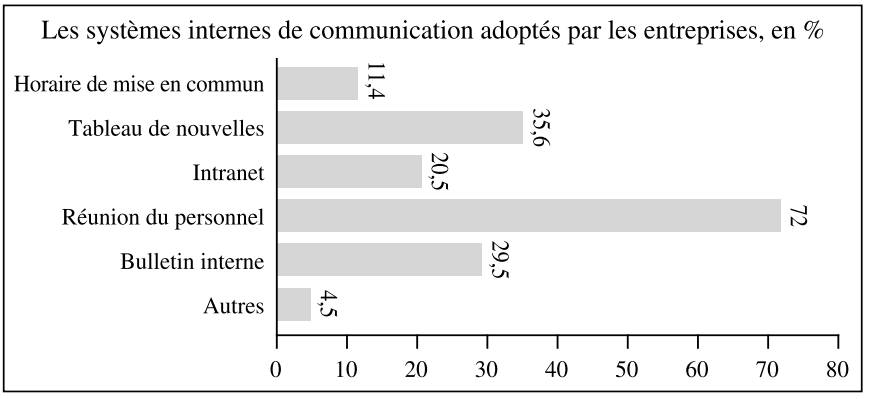

Les entreprises de notre échantillon adoptent divers systèmes de communication. Les réunions du personnel constituent le système le plus utilisé : $72 \%$ de ces entreprises s'en servent pour véhiculer de l'information à l'interne. Les entreprises qui comptent sur les tableaux de nouvelles à cette fin représentent $36 \%$. Un examen plus approfondi montre une variation significative entre les différents territoires. Par exemple, 35\% des entreprises implantées au Saguenay utilisent le bulletin interne comme système de communication, contre seulement 15,4\% à Rivière-du-Loup, 28\% à Manicouagan et 26,5\% dans la région de Québec.

De plus, les entreprises utilisent le tableau de nouvelles à proportion de $26,5 \%$ dans la région de Québec contre $24 \%$ des entreprises à Manicouaguan, $15 \%$ à Rivière-duLoup et $30 \%$ au Saguenay. Dans la région de Québec, $35 \%$ de ces entreprises utilisent l'Intranet comme système interne de communication. Ce pourcentage est de $20 \%$ au Saguenay alors qu'il ne dépasse pas $8 \%$ à Manicouagan et à Rivière-du-Loup. Il faut souligner cependant qu'une entreprise peut utiliser plusieurs systèmes de communication à la fois.

Les entreprises préfèrent davantage les réunions du personnel, les tableaux de nouvelles et les bulletins internes. Un pourcentage important de dirigeants d'entreprises recourt à I'Intranet.
Les entreprises préfèrent davantage les réunions du personnel, les tableaux de nouvelles et les bulletins internes. Un pourcentage important de dirigeants d'entreprises recourt à l'Intranet. On constate que les dirigeants d'entreprises utilisent plus l'Intranet dans la région de Québec que dans les autres territoires.

\subsection{Le classement de l'information}

L'avènement des nouvelles technologies impose de nouvelles façons de gérer les organisations. Des modifications significatives du savoir-faire s'imposent, et chaque organisation adopte un ou plusieurs systèmes de classement de l'information. Par rapport aux anciens, les nouveaux systèmes de classement de l'information bouleversent les habitudes et les fondements mêmes du savoir-faire entrepreneurial.

\section{Figure 9: Le classement de l'information, tout l'échantillon, en \%}

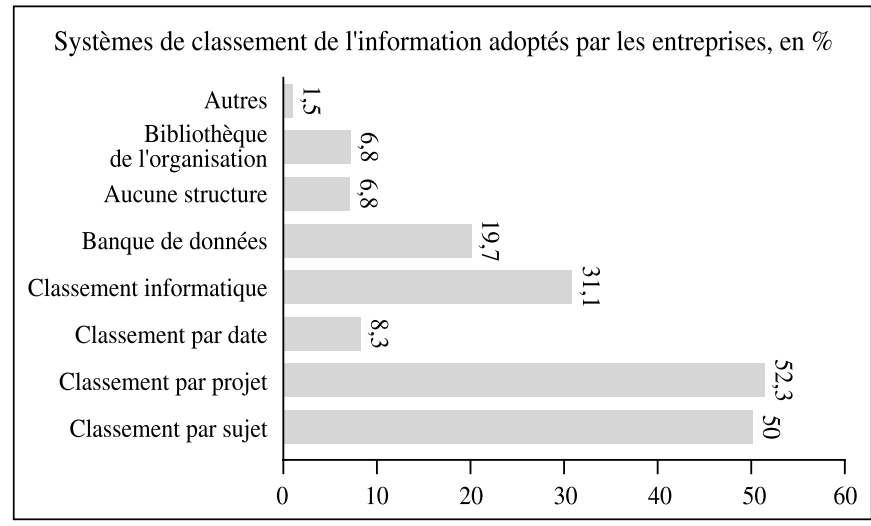

Le choix d'un système de classement de l'information constitue un indicateur de la capacité de l'entreprise à s'adapter aux vagues de changement apporté par les nouvelles technologies. Dans ce cadre, le questionnaire portait sur les différents systèmes de classement de l'information. La question était la suivante: De quelle façon classez-vous l'information reçue dans l'entreprise?

Le classement par sujet est adopté par $49 \%$ des entreprises de notre échantillon. À Manicouagan, le taux est de $55 \%$ alors qu'il est de $48 \%$ dans la région de Québec, $46 \%$ à Rivière-du-Loup et 32\% au Saguenay. De même, $39 \%$ des entreprises de notre échantillon optent pour le classement par projet. Ce pourcentage est de 53\% dans la région de Québec, de 68\% à Manicouagan, 43\% à Rivière-du-Loup et seulement 38,5\% au Saguenay. Le classement de l'information par date est adopté par seule- 
ment $8 \%$ des entreprises de notre échantillon. Cette catégorie représente $6 \%$ dans la région de Québec. Le pourcentage est un peu plus élevé à Manicouagan, où il atteint $12 \%$, alors qu'il ne dépasse pas $7 \%$ à Rivière-du-Loup.

Quant au classement informatique de l'information, il est utilisé par 44\% des entreprises de la région de Québec. Ce pourcentage dépasse ceux relevés au Saguenay (27\%), à Rivière-du-Loup (23\%) et à Manicouagan (28\%). C'est aussi dans la région de Québec que l'on a enregistré le pourcentage le plus élevé quant à l'utilisation des banques de données : $32 \%$ des entreprises de cette région utilisent ces banques, contre $16 \%$ à Manicouagan, $17 \%$ au Saguenay et seulement $8 \%$ à Rivière-du-Loup.

Les entreprises préfèrent le classement par sujet et par projet et l'on constate la montée du classement informatique et des banques de données. Le classement par sujet et par projet est plus important dans la région de Québec et au Saguenay qu'à Rivière-du-Loup et Manicouagan. Comparativement aux autres territoires, c'est dans la région de Québec qu'un pourcentage important d'entreprises recourt le plus au classement informatique de l'information et aux banques de données.

Donc, les entreprises préfèrent le classement par sujet et par projet et l'on constate la montée du classement informatique et des banques de données. Le classement par sujet et par projet est plus important dans la région de Québec et au Saguenay qu'à Rivière-du-Loup et Manicouagan. Comparativement aux autres territoires, c'est dans la région de Québec qu'un pourcentage important d'entreprises recourt le plus au classement informatique de l'information et aux banques de données.

\section{L'appropriation de la nouvelle technologie}

L'innovation technologique peut être comprise à partir de plusieurs indicateurs. Dans le cadre de cette enquête, le choix a été fixé sur le branchement des entreprises à l'Internet, l'utilisation de l'Internet, l'usage du Web, le soutien extérieur et l'usage du matériel informatique et des logiciels. À eux seuls, ces indicateurs montrent à quel point les entreprises s'approprient les nouvelles technologies et comment ces technologies parviennent à modifier les pratiques entrepreneuriales.

\subsection{Le branchement des entreprises à Internet}

On ne peut guère aborder l'innovation technologique sans parler du branchement à l'Internet. Si ce branchement est un indicateur important de cette même innovation, il est cependant difficile de voir comment il contribue à transformer le monde des affaires. Un moyen utile pour étudier cette innovation est la date de branchement des acteurs à l'Internet. La référence à cette date est susceptible d'aider à établir une certaine hiérarchie entre les quatre territoires retenus pour notre analyse.

On posait la question suivante: Depuis combien de temps êtes-vous branché à Internet? Les résultats obtenus montrent que $51,5 \%$ des entreprises de notre échantillon sont branchées à l'Internet depuis plus de deux ans. Cette catégorie est la plus importante dans la région de Québec (82\%) en comparaison à $43 \%$ au Saguenay, $40 \%$ à Manicouagan et seulement $31 \%$ à Rivière-du-Loup. On apprend aussi que $6 \%$ des entreprises de notre échantillon ne sont pas encore branchées à l'Internet. À Rivière-du-Loup, cette catégorie représente $23 \%$ des entreprises, contre seulement 5\% au Saguenay et $8 \%$ à Manicouagan.

Figure 10 : Le branchement des entreprises à l'Internet

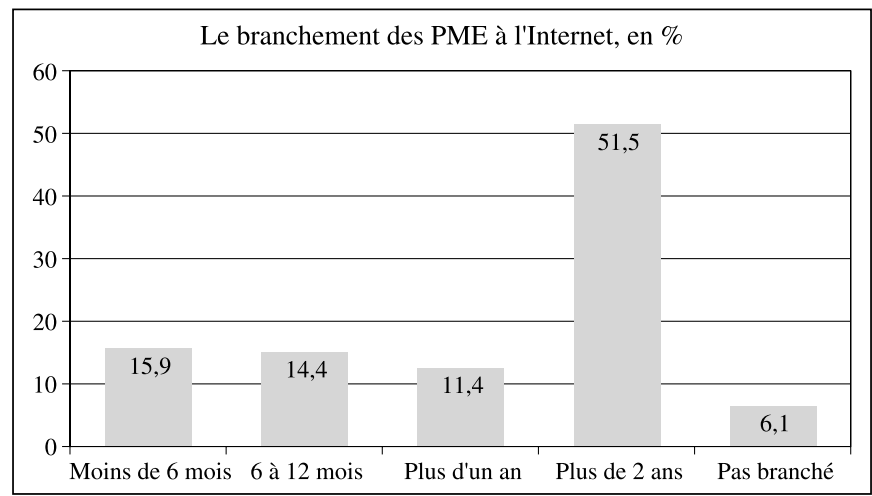

La majorité des entreprises sont branchées depuis plus de deux ans. Les entreprises

branchées à Internet depuis plus de deux ans sont plus importantes dans la région de Québec que dans les autres territoires. Les entreprises qui ne sont pas encore branchées

à Internet sont plus nombreuses à Rivièredu-Loup que dans les autres territoires. 
On constate que la majorité des entreprises sont branchées depuis plus de deux ans. Les entreprises branchées à Internet depuis plus de deux ans sont plus importantes dans la région de Québec que dans les autres territoires. Les entreprises qui ne sont pas encore branchées à Internet sont plus nombreuses à Rivière-du-Loup que dans les autres territoires.

\subsection{Utilisation d'Internet?}

Le taux d'utilisation d'Internet par les entreprises a ceci d'intéressant qu'il constitue un autre indicateur de l'innovation technologique. Il faut donc chercher à comprendre comment cet usage se manifeste dans les différents territoires. La question était la suivante: Quelles sont les utilisations que vous effectuez à l'aide d'Internet?

L'Internet sert à $86 \%$ des entreprises de notre échantillon pour l'envoie de courriels. Au Saguenay et à Québec, ce pourcentage est très élevé: il y atteint respectivement $97 \%$ et $91 \%$. Le pourcentage est de $88 \%$ à Manicouagan et de $77 \%$ à Rivière-du-Loup. On peut donc dire que le recours au courriel est l'une des principales tâches que ces organisations font par Internet. De même, 67\% utilisent Internet pour acquérir des données stratégiques. Le pourcentage est de 73,5\% dans la région de Québec et de $73 \%$ au Saguenay. Il est relativement faible à Manicouagan $(52 \%)$ et à Rivière-du-Loup (46\%).

\section{Figure 11: L'usage de l'Internet, tout l'échantillon}

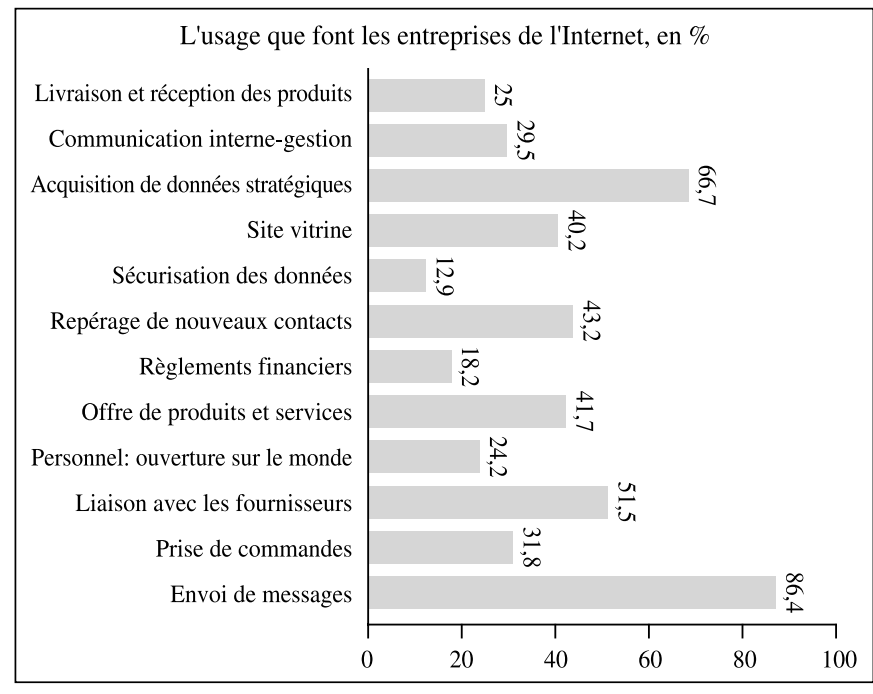

Les entreprises des quatre territoires utilisent d'abord Internet pour envoyer des courriels. Elles l'utilisent aussi pour acquérir des données, mais cette catégorie est plus importante dans la région de Québec et au Saguenay qu'à
Manicouagan et à Rivière-du-Loup. Les entreprises implantées dans la région de Québec et au Saguenay utilisent plus Internet pour établir des liaisons avec les fournisseurs, livrer et recevoir des produits et à des fins personnelles.

\section{Les entreprises des quatre territoires utilisent d'abord Internet pour envoyer des courriels. Elles l'utilisent aussi pour acquérir des données, mais cette catégorie est plus importante dans la région de Québec et au Saguenay qu'à Manicouagan et à Rivière-du-Loup. Les entre- prises implantées dans la région de Québec et au Saguenay utilisent plus Internet pour établir des liaisons avec les fournisseurs, livrer et recevoir des produits et à des fins personnelles.}

Par ailleurs, seulement $20 \%$ des entreprises implantées à Manicouagan utilisent l'Internet pour les prises de commandes. Ce pourcentage est de $23 \%$ à Rivière-du-Loup, $30 \%$ au Saguenay et atteint $47 \%$ dans la région de Québec. L'ensemble de ces résultats montre aussi que $65 \%$ des entreprises de la région de Québec profitent de l'Internet pour établir des liaisons avec les fournisseurs. Dans cette même région, $41 \%$ s'en servent pour livrer et recevoir des produits. Internet est utilisé par $47 \%$ des dirigeants de ces organisations à des fins personnelles et d'ouverture sur le monde. Le Saguenay vient en deuxième place, avec des pourcentages qui sont respectivement de $52 \%, 25 \%$ et $20 \%$.

\subsection{L'usage du Web et de son potentiel}

La troisième question posée dans le cadre de cette section consacrée à l'innovation technologique porte sur l'usage du Web dans le domaine commercial. Plus précisément, on voulait savoir si les entreprises faisaient des achats sur le Web et si elles utilisent suffisamment son potentiel. On posait les deux questions suivantes: Faites-vous des achats sur le Web? Considérez-vous que vous utilisez suffisamment le potentiel du commerce électronique?

\section{Figure 12: L'achat et l'exploitation du potentiel} du Web, en \%

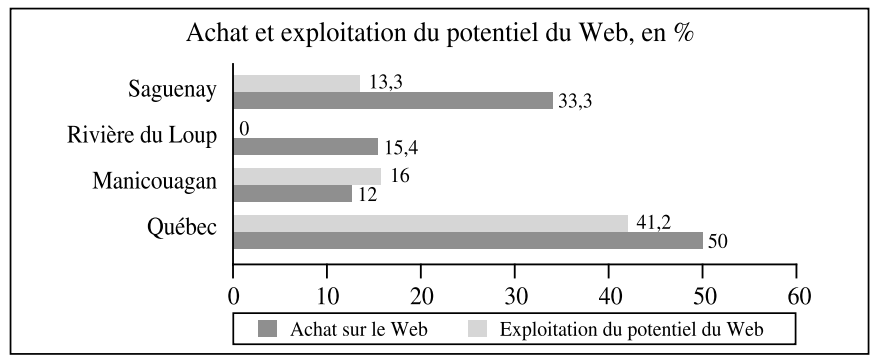


On constate que $32 \%$ des entreprises de notre échantillon font des achats sur le Web. Le pourcentage est plus élevé dans la région de Québec (50\%). Il ne dépasse pas $33 \%$ au Saguenay et s'établit à 15\% à Rivière-du-Loup et à seulement $12 \%$ à Manicouagan. Par ailleurs, seulement $20 \%$ des entreprises affirment exploiter suffisamment le potentiel du Web. Ce pourcentage est de $41 \%$ dans la région de Québec, $16 \%$ à Manicouagan et $13 \%$ au Saguenay.

Les entreprises implantées dans la région de Québec et au Saguenay utilisent davantage le Web pour faire des achats. Un faible pourcentage des entreprises sondées exploite le potentiel du Web alors qu'un grand pourcentage des entreprises de Québec n'hésite pas à le faire.

\subsection{Le soutien extérieur}

Pour plusieurs entreprises, l'innovation technologique exige des compétences qui ne sont pas toujours à leur portée. Souvent, elles cherchent à combler le manque d'une main-d'œuvre qualifiée par le recours à des ressources extérieures. Nous avons essayé d'identifier la nature du soutien extérieur recherché par les entreprises implantées dans les quatre territoires retenus pour notre analyse. La question était la suivante: À quels soutiens extérieurs l'entreprise peut-elle recourir pour des questions reliées aux technologies de l'information?

Les réponses recueillies permettent de constater que 52\% des entreprises de notre échantillon utilisent les services offerts par les conseillers et les fournisseurs en matériel et logiciels, $29 \%$ profitent des services offerts par les fournisseurs d'accès à l'Internet et $22 \%$ recourent aux services que proposent les créateurs de pages Web.
On constate donc que les entreprises des quatre territoires sollicitent davantage les services des conseillers et fournisseurs en matériel informatique et logiciels et des fournisseurs d'accès à Internet. De plus, il n'y a pas de différence significative entre les quatre territoires.

\subsection{Matériel informatique et logiciels}

L'usage du matériel informatique et d'un nombre de logiciels est un autre indicateur important de l'innovation technologique. Les composantes informatiques sont nombreuses, variées et servent à de nombreuses tâches liées à la gestion des organisations. Le matériel et les logiciels se développent rapidement, incitant l'organisation à s'efforcer de mieux s'adapter aux changements qui en résultent. Généralement, les résultats sont considérables, surtout quand les dirigeants ont choisi le matériel et les logiciels qui répondent adéquatement aux besoins ressentis à l'intérieur de l'organisation. Cependant, le développement du secteur informatique et des logiciels est tellement rapide que plusieurs entreprises ne parviennent pas à suivre les vagues de changement.

L'usage du matériel informatique et d'un nombre de logiciels est un autre indicateur important de l'innovation technologique. Les composantes informatiques sont nombreuses, variées et servent à de nombreuses tâches liées à la gestion des organisations. Le matériel et les logiciels se développent rapidement, incitant l'orga-

\section{Figure 13: Le soutien extérieur recherché par les entreprises, en \%}

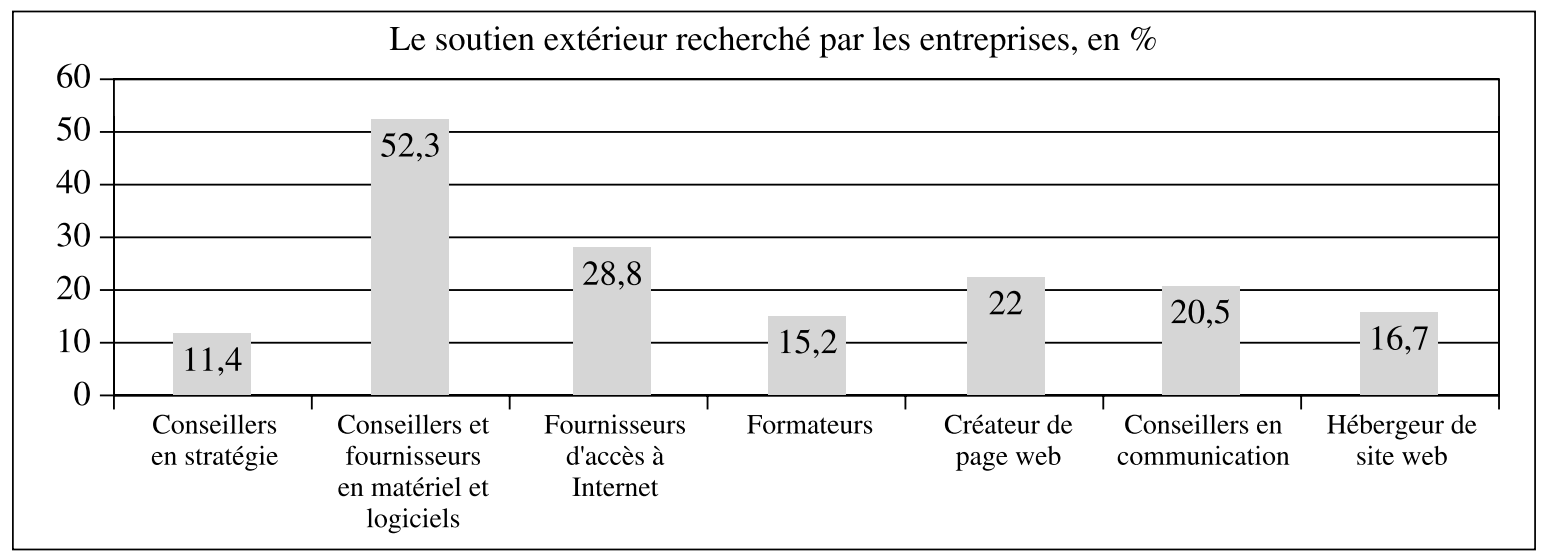


nisation à s'efforcer de mieux s'adapter aux changements qui en résultent. Généralement, les résultats sont considérables, surtout quand les dirigeants ont choisi le matériel et les logiciels qui répondent adéquatement aux besoins ressentis à l'intérieur de l'organisation. Cependant, le développement du secteur informatique et des logiciels est tellement rapide que plusieurs entreprises ne parviennent pas à suivre les vagues de changement. Il faut donc chercher à voir l'intérêt pour notre enquête de comprendre comment ces changements modifient les pratiques entrepreneuriales.

En fait, la transformation du matériel informatique est «le principal vecteur par lequel le paradigme informationnel et le processus de globalisation agissent sur la société dans son ensemble ${ }^{7}$ ». On peut même dire que l'usage de ce matériel et de ces logiciels influence la prise de décision au sein de toute organisation. En effet, une décision «s'observe à travers le constat d'un changement. Ce qui est observable, c'est «en aval» du changement, des passages à l'action, c'est-à-dire des pratiques, et «en amont» des représentations qui structurent les formes du passage à l'action et de la décision ${ }^{8} »$.

Quoi qu'il en soit, les composantes technologiques qui nous intéressent se placent au centre même des décisions et des actions entrepreneuriales. C'est pourquoi la question posée a été formulée en ces termes: Quel est le niveau de pénétration de chacune des technologies de l'information dans votre entreprise?

On constate que les composantes du matériel informatique qui sont opérationnelles dans les entreprises sont: le télécopieur, l'ordinateur et les autres imprimantes. Le télécopieur est utilisé dans $95 \%$ des entreprises. Le pourcentage est de $92 \%$ dans le cas des ordinateurs. De même, les autres imprimantes sont utilisées dans $96 \%$ des entreprises de notre échantillon. Par ailleurs, le pourcentage des entreprises qui n'utilisent pas de télécopieur et d'autres imprimantes ne dépasse pas 3\%. Dans le cas des ordinateurs, ce pourcentage est de 6\%. D'un autre côté, les composantes de l'équipement informatique qui sont moins utilisées sont les tables traçantes, qui n'existent pas dans $61 \%$ des entreprises québécoises, le CAIO (37\%), le lecteur optique $(55 \%)$ et les robots $(69 \%)$.

Dans un deuxième temps, nous nous sommes intéressés aux logiciels utilisés par les entreprises. Les logiciels de traitement de texte sont utilisés dans $92 \%$ des entreprises de l'échantillon. Ce pourcentage est de $93 \%$ dans le cas des logiciels de comptabilité. Il est de $78 \%$ pour ce qui est des logiciels de traitement de bases de données. Les logiciels de gestion d'inventaire sont utilisés dans 58\% des cas. Par ailleurs, 53\% des entreprises n'ont pas de logiciels servant de système expert. De même, il y a absence de logiciels d'échange d'information informatisée dans $50 \%$ des cas. Quant aux logiciels de gestion du personnel, ils sont absents chez $35 \%$ des entreprises; enfin, le taux est de $14 \%$ en ce qui concerne les logiciels de gestion des bases de données.

Les entreprises s'approprient davantage les télécopieurs, les ordinateurs et les autres imprimantes. Elles s'approprient moins le matériel qui exige un très haut degré de spécialisation, par exemple, le CAIO, le lecteur optique et les robots. Il n'y aucune différence entre les quatre territoires. Les entreprises s'approprient beaucoup les logiciels de base (traitement de texte, traitement de données, etc.) et elles s'approprient moins les logiciels trop sophistiqués.

\section{Conclusion}

L'usage des nouvelles technologies et en particulier l'informatisation compte pour beaucoup dans le succès de certains territoires. C'est sur cet aspect que notre recherche a tenté de jeter un peu de lumière, en prenant pour sujet d'étude, quatre territoires du Québec. L'analyse des résultats de notre recherche suggère que deux logiques différentes influencent l'appropriation du matériel informatique et des logiciels. La première relève de l'usage d'un matériel relativement traditionnel (comme le téléphone et la télécopie), qu'on adopte communément à des besoins créés principalement par le développement rapide de l'ordre technologique; la seconde est fondée sur l'usage d'une très haute technologie et rend imprévisibles des comportements nouveaux, variant d'un territoire à l'autre, et même parfois d'une catégorie d'acteurs à une autre. Ces deux logiques doivent certainement influencer inégalement le développement économique, social et culturel.

Alors que la conjoncture a développé de nouvelles pratiques entrepreneuriales, tout territoire offre à l'acteur la possibilité de développer des formes complexes de rapports aux composantes technologiques. Jamais identiques, ces rapports stimulent les acteurs en quête d'efficacité et de performance, alors que pour d'autres, ils sont un obstacle. Des conditions conjoncturelles et structurelles font que ces rapports apparaissent, se modifient et prennent de l'importance ou se dégradent. C'est que les acteurs, n'en voyant pas au même degré la nécessité, ne fournissent pas les mêmes efforts pour intégrer les connaissances scientifiques et le savoir-faire associés aux nouvelles technologies. 


\section{Dans un territoire, la régression des anciens modes de fonctionnement des entreprises se traduit d'ordinaire par des pratiques et des comportements qui marginalisent les pouvoirs traditionnels. À l'opposé, des horizons nouveaux s'ouvrent devant les acteurs qui réussissent à s'insérer dans un nouvel environnement dont ils tirent profit pour déve- lopper leur organisation et leur territoire.}

Dans un territoire, la régression des anciens modes de fonctionnement des entreprises se traduit d'ordinaire par des pratiques et des comportements qui marginalisent les pouvoirs traditionnels. À l'opposé, des horizons nouveaux s'ouvrent devant les acteurs qui réussissent à s'insérer dans un nouvel environnement dont ils tirent profit pour développer leur organisation et leur territoire. Si l'on regarde d'un peu plus près, il semble clair que c'est principalement l'informatisation et la façon dont elle est utilisée qui fait la différence. Bouchy constate que nos sociétés

«sont marquées par une profonde mutation économique et sociologique de l'ingénierie des systèmes d'information. Bien sûr la technique continue à évoluer, la miniaturisation progresse encore, les prix des matériels baissent régulièrement, la pénurie d'informaticiens compétents perdure et l'informatique supprime toujours beaucoup plus d'emplois ici qu'elle en crée là, ou tout au moins accélère une mutation des emplois orientés vers le tertiaire et les services. Cependant, comme toute révolution industrielle le permet, l'informatique innerve tous les aspects de la vie quotidienne et sans rupture, de la vie familiale à la vie professionnelle, la collectivité est entrée dans un monde où l'informatique devient l'outil privilégié de l'activité des entreprises et de la communication des hommes ${ }^{9} »$.

Les résultats de notre enquête montrent que l'offre, la demande et le traitement de l'information sont interreliés ils s'inscrivent dans une stratégie globale d'action-réaction aux contraintes et possibilités offertes par le nouvel ordre technologique. Des trajectoires différentes entre les entreprises et entre les territoires amènent une informatisation graduée selon les besoins ressentis, la vision des acteurs et l'interpénétration des systèmes informatiques et des systèmes d'information.

Il apparaît en effet que les systèmes informatiques et les systèmes d'information se développent en parallèle, mais pas au même rythme, parce qu'utilisés différemment par les entreprises dans leurs stratégies d'affaires selon la nature des activités économiques dominantes et, probablement, selon les formes de socialisation en place.

L'offre, la demande et le traitement de I'information sont interreliés - ils s'inscrivent dans une stratégie globale d'action-réaction aux contraintes et possibilités offertes par le nouvel ordre technologique. Des trajectoires différentes entre les entreprises et entre les territoires amènent une informatisation graduée selon les besoins ressentis, la vision des acteurs et l'interpénétration des systèmes informatiques et des systèmes d'information.

Les résultats de notre enquête montrent aussi qu'en territoire métropolitain, où l'on intègre facilement divers systèmes d'information, les entreprises utilisent davantage divers moyens de communication en mettant l'accent sur les moyens fondés sur une technologie de pointe, alors que dans un territoire périphérique, les moyens traditionnels de communication sont surexploités et qu'on $\mathrm{y}$ adopte des systèmes de traitement de l'information adaptés à un savoir-faire qui a traditionnellement fait ses preuves (classement d'information par sujet et par projet). On y voit deux formes de socialisation et de savoir-faire: l'une évoluant en harmonie avec les exigences du nouvel ordre technologique, l'autre s'en méfiant jusqu'à le rejeter. La coexistence de ces deux formes de socialisation autorise toute initiative favorisant l'automatisation de diverses tâches, accentuant de ce fait des disparités entre les territoires métropolitains et périphériques, mais sans que ces disparités aboutissent au déclin de ces derniers.

On peut toutefois avancer que ces disparités incitent les entreprises implantées dans les territoires périphériques à récupérer le savoir et le savoir-faire traditionnellement utilisés par les entreprises implantées dans les territoires métropolitains. Cette récupération s'accompagne d'une tentative de rattrapage de la part des entreprises implantées dans les territoires périphériques qui essaient de s'approprier certains objets de haute technologie. Dans ce cas, l'appropriation de matériel et de logiciels a des effets importants puisque «l'automatisation des tâches permet de concentrer les efforts de production des professionnels sur les tâches à forte valeur ajoutée, d'assurer la 
qualité des procédés et des produits et de produire dans des conditions de coûts et de délais connues à l'avan$\mathrm{ce}^{10}{ }^{1}$. L'absence d'économie d'échelle et d'économie d'agglomération dans les territoires périphériques de même que les coûts élevés de transport vers les grands marchés peuvent minimiser les effets de l'automatisation mais n'affectent pas ou peu les différentes formes de proximité qui peuvent être encouragées par les NTIC.

Ces disparités incitent les entreprises implantées dans les territoires périphériques à récupérer le savoir et le savoir-faire traditionnellement utilisés par les entreprises implantées dans les territoires métropolitains. Cette récupération s'accompagne d'une tentative de rattrapage de la part des entreprises implantées dans les territoires périphériques qui essaient de s'approprier certains objets de haute technologie.

Dans certains territoires, les entreprises n'adoptent pas facilement les nouvelles technologies, soit parce qu' elles ne disposent pas d'une main d'œuvre qualifiée, soit parce que la conjoncture économique et sociale ne s'y prête pas. Toute appropriation du matériel informatique et des logiciels s'inscrit dans un cheminement entrepreneurial long. Une dynamique technologique devient favorable au développement d'un territoire, qu'il soit métropolitain ou périphérique, si les entreprises sont à même, au moment opportun, d'identifier collectivement et individuellement les composantes technologiques qui répondent adéquatement à leurs besoins immédiats. Les réseaux d'entreprises et les autres formes d'interaction choisies par les dirigeants offrent le cadre général qui favorise cette identification. Cependant, le choix des composantes technologiques se fait en général individuellement et ne doit pas nuire à la vocation initiale et à l'identité propre de l'entreprise et du territoire. C'est à cette condition que l'adoption des nouvelles technologies peut-être une source d'innovation.

L'usage des technologies de pointe précipite l'abandon des anciennes pratiques; parfois d'anciens objets se dotent de nouvelles vocations qu'on ne peut exploiter sans une maîtrise au moins partielle des principales composantes de la haute technologie. Quel que soit son territoire, l'entrepreneur doit apprendre comment s'assurer d'un meilleur passage d'un monde à l'autre, ce qui constitue en soi un acte d'innovation.
Comme les inventeurs, les industriels cherchent à mettre sur le marché des produits intéressants par leur beauté, leur performance, etc. Ils exposent avec fierté des innovations qui projettent une image de marque favorable de leur firme ${ }^{11}$. Il y a ainsi existence concomitante d'anciens et de nouveaux produits, marquant l'abandon de procédés en déclin pour une utilisation en progression des nouvelles technologiques d'information.

Une plus grande émergence de pratiques nouvelles et innovatrices dans un territoire métropolitain qu'en périphérie s'explique par les objets à haute technologie. Bien qu'ils améliorent le savoir-faire des entrepreneurs, quels que soient leurs territoires d'implantation, plusieurs objets sophistiqués sont plus souvent utilisés dans les métropoles que dans les régions périphériques. Ils intègrent mieux le mode de vie urbain, davantage ouvert sur des dynamiques internationales. Il reste toutefois qu'une appropriation inégale de l'informatique a une incidence importante sur ces disparités. Par exemple, alors qu'une technologie est en perpétuel renouvellement, l'exécution de tâches complexes par des employés peu formés constitue un défi majeur que les entreprises affrontent régulièrement. Le recours à des conseillers en matériel informatique et logiciels comme soutien extérieur constitue une solution que plusieurs adoptent pour se tenir à jour.

\section{Une plus grande émergence de pratiques nouvelles et innovatrices dans un territoire métropolitain qu'en périphérie s'explique par les objets à haute technologie. Bien qu'ils améliorent le savoir-faire des entrepreneurs, quels que soient leurs territoires d'implantation, plusieurs objets sophistiqués sont plus souvent utilisés dans les métropoles que dans les régions périphériques. Ils intègrent mieux le mode de vie urbain, davantage ouvert sur des dynamiques internationales. II reste toutefois qu'une appropriation inégale de l'informatique a une incidence importante sur ces disparités.}

\section{Notes et références}

1 Lahcen Darhouani est professionnel de recherche. Il termine actuellement son doctorat en Développement régional à l'UQAC. Cette étude fut réalisée grâce à la collaboration de Ali Doubi et Josée Gauthier, sous la direction du Professeur Marc-Urbain Proulx. 
2 La même entreprise peut avoir de la concurrence à plusieurs niveau en même temps.

3 Castells, M., La société en réseau, l'ère de l'information, Fayard, c1998, p. 42-43, note 34

4 Ibid., p. 39

5 Ibid

6 Ibid., p. 22.

7 Ibid., p. 239.

8 Vinokur, Annie, (dir), Les décisions économiques, Paris, Économica, 1998, p. 357-358.

9 Bouchy, Serge, L'ingénierie des systèmes d'information évolutifs, Paris, Eyrolles, (Col. Informatique pratique dans les entreprises), 1994, p. 25.

10 Ibid., p. 142
11 Par exemple, dans une revue de vulgarisation informatique, le centre de développement logiciel Motorola de Montréal, (MCSC) publiait un communiqué annonçant sa position au sommet canadien de la production logicielle: «le Motorola a annoncé que son MSCS, inauguré en 1999, avait atteint le plus haut niveau de maturité du modèle SEI-CMM (Software Engineering Institute, Capability Maturity Model; www.sei.cmu.edu). L'évaluation a été effectuée à partir de la méthode CMM très stricte d'amélioration des processus internes (CBA-IPI). C'est la première fois qu'un organisme canadien atteint le niveau 5 du SEI-CMM. Le niveau 5 est décerné aux organismes qui affichent une amélioration continue et quantifiable de leurs processus logiciels... Les organismes doivent aussi être axés sur les idées innovatrices. Le niveau 5 est un résultat qu'atteignent rarement quelques centres de R\&D nord-américain. ... La mission du MCSC est de faire de la recherche et développement des éléments de base utilisés dans les futures dispositifs d'appareils de communication avec capacité multimédia et dans les nouvelles applications de haute technologie...»; Revue Québecmicro, www.quebecmicro.com, mars 2002, p. 10. 